\title{
Electron transport across electrically switchable magnetic molecules
}

\begin{abstract}
Sujeet K. Shukla* and Stefano Sanvito ${ }^{\dagger}$
School of Physics and CRANN, Trinity College, Dublin 2, Ireland

(Received 11 May 2009; revised manuscript received 2 November 2009; published 30 November 2009)

We investigate the electron-transport properties of a model magnetic molecule formed by two magnetic centers whose exchange coupling can be altered with a longitudinal electric field. In general, we find a negative differential conductance at low temperatures originating from the different scattering amplitudes of the singlet and triplet states. More interestingly, when the molecule is strongly coupled to the leads and the potential drop at the magnetic centers is only weakly dependent on the magnetic configuration, we find that there is a critical voltage $V_{\mathrm{C}}$ at which the current becomes independent of the temperature. This corresponds to a peak in the low-temperature current noise. In such limit, we demonstrate that the quadratic current fluctuations are proportional to the product between the conductance fluctuations and the temperature.
\end{abstract}

DOI: 10.1103/PhysRevB.80.184429

PACS number(s): 72.25.Rb, 73.22.-f, 75.30.Wx, 75.50.Xx

\section{INTRODUCTION}

An intriguing aspect of electronic transport is the interaction between the current electrons and the internal degrees of freedom of the conductor. Atomic positions and vibrations are certainly at the research center stage, electromigration being the most obvious example of interplay between the current and the atoms motion. The situation becomes even more intriguing at the nanoscale, where quantized vibrations can be detected by measuring the electron current and its derivatives with respect to the applied bias. This is the principle of inelastic electron-tunneling spectroscopy (IETS). Furthermore also the reverse effect is possible, namely, one can control the atomic positions of a nano-object by exciting appropriately some vibrational modes. Current-induced chemical reactions ${ }^{1}$ and nanocatalysis ${ }^{2}$ on surfaces are among the most appealing potential applications of this field.

Equally important is the interplay between the electron current and the magnetic texture of a magnetic device. Such an interplay underpins the giant magnetoresistance effect $^{3}$ and its reverse, i.e., current-induced magnetization dynamics. ${ }^{4}$ Considerably less investigated are the same phenomena at the atomic scale. This is mainly due to the intrinsic difficulties of both manipulating and detecting a few spins. In addition, magnetic excitations occur at energies lower than those involved in molecular vibrations so that the measuring temperatures are often rather low. Still there are notable examples, such as ultra low-temperature IETS of magnetic atoms on surfaces ${ }^{5}$ and of two-probe devices incorporating single magnetic molecules. ${ }^{6}$

An exciting prospect for scaling down spin dynamics to the atomic level may be given by the ability of manipulating the magnetic configuration of a molecule with an electric potential instead of an electric current. Electrically induced alteration of the exchange coupling has been already predicted for two-centers magnetic molecules ${ }^{7}$ and molecular nanowires, ${ }^{8}$ and it is essentially based on the fact that the Stark shift of a magnetic object may depend on its magnetic state. This effect can be a crucial ingredient for the physical implementation of quantum computing based on spins. ${ }^{9,10}$

An intriguing question is whether or not the dependence of the exchange coupling over an electrical potential in a magnetic molecule can be detected electrically. This is the goal of our paper where we investigate the current-voltage, $I-V$, curve of a two-terminal device incorporating a twocenter magnetic molecule in which the exchange coupling changes with bias. Importantly we find that, in particular conditions of coupling between the molecule and the electrodes, there is a critical voltage $V_{\mathrm{C}}$ at which the current becomes independent of the temperature. This is accompanied by a negative differential conductance (NDC) at low temperature originating from the difference in scattering amplitude of the different spin states of the molecule.

\section{SYSTEM SETUP AND COMPUTATIONAL METHOD}

In Fig. 1 we show the simple model system investigated, which comprises a diatomic magnetic molecule sandwiched between two one-dimensional (1D) nonmagnetic electrodes. The system is described by the $s-d$ model, ${ }^{11,12}$ where spin of the current carrying $s$ electrons is exchange coupled to the local spins $S_{1}$ and $S_{2}(d)$ of the two atoms in the dimer. $S_{1}$ and $S_{2}$ are treated as classical variables and their orientation determines the scattering potential for the $s$ electrons. These are described by a tight-binding Hamiltonian with a single $s$ orbital per site at half filling. The on-site energy and hopping integral in the electrodes are $\epsilon_{\mathrm{L}}=2 \mathrm{eV}$ and $\gamma_{\mathrm{L}}=-2 \mathrm{eV}$, a

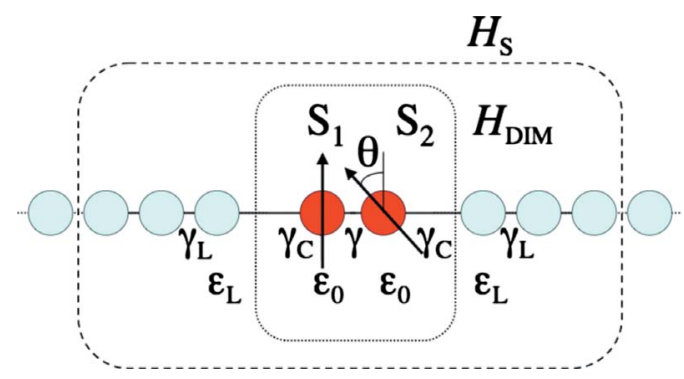

FIG. 1. (Color online) The model system investigated: a dimer of magnetic atoms, dark gray (red online), carrying, respectively, spin $S_{1}$ and $S_{2}$, is attached to two 1D nonmagnetic electrodes, light gray (light blue online). The Hamiltonian for the dimer is $H_{\text {DIM }}$ The scattering region (dashed box) includes the dimer and six atoms of the electrodes and it is described by the Hamiltonian matrix $H_{\mathrm{S}}$. 
choice which maintains the system far from the van Hoof singularities at any voltage investigated. No local spins are present in the electrodes so that their electronic structure is not spin polarized. The Hamiltonian of the dimer is

$$
H_{\mathrm{DIM}}=\sum_{\alpha \beta} \sum_{i}^{1,2} h_{i}^{\alpha \beta} c_{i}^{\alpha \dagger} c_{i}^{\beta}+\sum_{\sigma} \gamma\left(c_{1}^{\sigma \dagger} c_{2}^{\sigma}+c_{2}^{\sigma \dagger} c_{1}^{\sigma}\right),
$$

where $h_{i}^{\alpha \beta}$ is the on-site Hamiltonian matrix of the $i$ th atom of the dimer, $\gamma$ is the hopping parameter, and $c_{i}^{\sigma \dagger}\left(c_{i}^{\sigma}\right)$ is the creation (annihilation) operator for an electron with spin $\sigma(\uparrow, \downarrow)$ at the site $i$. We have defined $h_{i}^{\alpha \beta}=\left[\epsilon_{0}+U\left(\rho_{i}\right.\right.$ $\left.\left.-\rho_{0}\right)\right] \delta_{\alpha \beta}-J_{\mathrm{sd}} \vec{S}_{i} \cdot(\vec{\sigma})_{\alpha \beta}$, where $\vec{\sigma}$ are the Pauli matrices, $\rho_{i}$ is the total occupation of $i$ th site, $\rho_{0}=1$ is the site occupation in the neutral configuration, $U=1 \mathrm{eV}$ is the atomic charging energy and $J_{\mathrm{sd}}=2 \mathrm{eV}$ is the exchange parameter between the $s$ electrons and the local spins. In our calculations we consider $\epsilon_{0}=2 \mathrm{eV}, \gamma=-0.1 \mathrm{eV}$, and $\left|\vec{S}_{i}\right|=1$. In absence of spinorbit interaction and spin polarization of the electrodes the scattering potential is determined only by the mutual angle, $\theta$, between the two local spins. Finally, the dimer and the electrodes are coupled by the hopping integral $\gamma_{\mathrm{C}}$. In particular we explore the two cases in which $\gamma_{\mathrm{C}}=1 / 4 \gamma_{\mathrm{L}}$ and $\gamma_{\mathrm{C}}$ $=1 / 2 \gamma_{\mathrm{L}}$. These parameters are only illustrative and have been chosen in order to maximize the difference in conductance between different spin states of the molecule ( $\theta=0$ vs $\theta=\pi)$.

The nonequilibrium Green's function method ${ }^{13}$ applied to our tight-binding Hamiltonian ${ }^{14}$ is used to calculate the transport properties. The central quantity is the retarded Green's function of the scattering region

$$
G(E)=\lim _{\eta \rightarrow 0+}\left[(E+i \eta)-H_{\mathrm{DIM}}-\Sigma_{\mathrm{L}}-\Sigma_{\mathrm{R}}\right]^{-1},
$$

where $E$ is the energy, $H_{\mathrm{S}}$ is the Hamiltonian matrix of the scattering region, and $\Sigma_{L}\left(\Sigma_{R}\right)$ is the self-energy of the left(right-)hand side electrode. This latter describes the interaction between the scattering region, which includes the dimer and six atoms of the electrodes (see Fig. 1), and the electrodes. $G(E)$ enters in a self-consistent procedure to evaluate the stationary occupation of the scattering region and once convergence is achieved the two-probe microscopic current, $i(V)$, at the voltage $V$ is extracted from the Landauer formula. ${ }^{14}$

Since at any given temperature the angle between the magnetic moments in the dimer fluctuates, for any microscopic quantity $q$ we can define its macroscopic counterpart, $Q$, as the thermal average over all the possible angles

$$
Q(V)=\langle q(V)\rangle=\frac{\int_{\mathcal{E}_{\min }}^{\mathcal{E}_{\max }} q(\theta, V) \mathrm{e}^{-\mathcal{E}_{12} / k_{\mathrm{B}} T} \mathrm{~d} \mathcal{E}}{\int_{\mathcal{E}_{\min }}^{\mathcal{E}_{\max }} \mathrm{e}^{-\mathcal{E}_{12} / k_{\mathrm{B}} T} \mathrm{~d} \mathcal{E}}
$$

thus that if $q=i$ one obtains the macroscopic current, $I$. Here $\mathcal{E}_{12}$ is the dimer magnetic energy, which writes

$$
\mathcal{E}_{12}=-J_{\mathrm{dd}} \cos \theta, \quad J_{\mathrm{dd}}=a+b \quad v_{\mathrm{d}}^{2}(\theta, V)
$$

and $\mathcal{E}_{\text {min }}\left(\mathcal{E}_{\max }\right)$ is its minimum (maximum) value. In the Eqs. (4) above $J_{\mathrm{dd}}$ is the exchange energy between the two spins, which in turns is a quadratic function of the electrical poten-

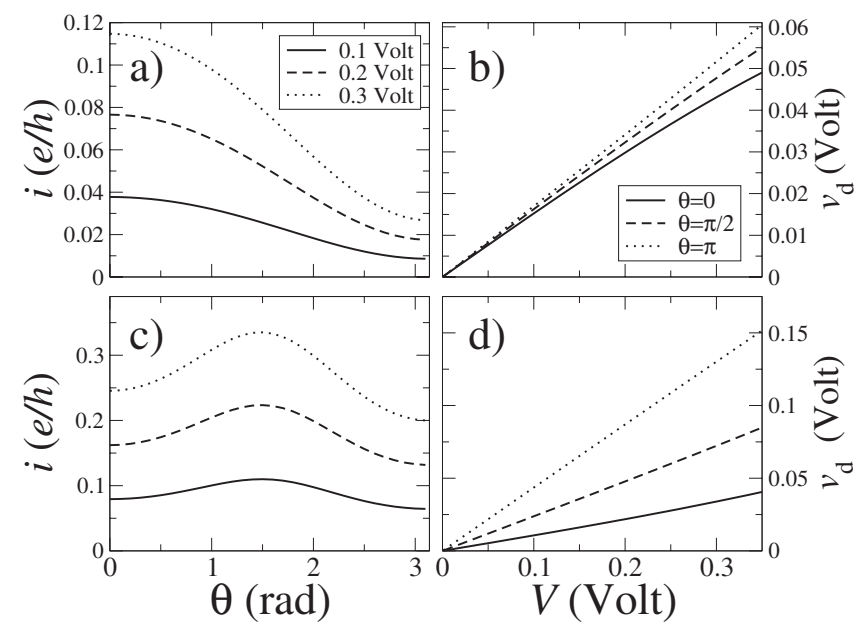

FIG. 2. Microscopic transport quantities. The microscopic current, $i$, as a function of the angle, $\theta$, is shown in panels (a) and (c) for different voltages $V$. Panels (b) and (d) show the internal potential drop, $v_{\mathrm{d}}$, as a function of the external bias and for different angles. Panels (a) and (b) are for $\gamma_{\mathrm{C}}=1 / 4 \gamma_{\mathrm{L}}$ while (c) and (d) are for $\gamma_{C}=1 / 2 \gamma_{L}$.

tial difference between them, $v_{\mathrm{d}}$. This latter is an intrinsic function of both $V$ and $\theta$. Finally the constants $a$ and $b$ are fixed to the values of $a=0.001 \mathrm{eV}$ and $b=-0.8 \mathrm{eV} / \mathrm{V}^{2}$. Note that the functional dependence of $J_{\mathrm{dd}}$ over $v_{\mathrm{d}}$ implies a critical voltage at which the exchange energy changes sign, i.e., the magnetic coupling turns from ferromagnetic to antiferromagnetic. ${ }^{7,8}$

\section{RESULTS AND DISCUSSION}

We begin our analysis by investigating the microscopic quantities, i.e., the current $i(V, \theta)$ and the dimer internal potential drop $v_{\mathrm{d}}$. In Fig. 2 we present, for both choices of coupling $\gamma_{\mathrm{C}}, i-\theta$ for different voltages and $v_{\mathrm{d}}-V$ for different angles $\theta$. In the case of $\gamma_{\mathrm{C}}=1 / 4 \gamma_{\mathrm{L}}$ the current varies as $i(V, \theta) \sim\left[i_{0}+i_{1} \cos \theta\right] V$ with $i_{0}$ and $i_{1}$ two constants. At the same time $v_{\mathrm{d}}$ is only weakly dependent on the internal spin configuration, i.e., the $v_{\mathrm{d}^{-}} V$ curve changes little with the angle $\theta$ [Fig. 2(b)]. In contrast for $\gamma_{\mathrm{C}}=1 / 2 \gamma_{\mathrm{L}}$ the current peaks at approximately $\theta=\pi / 2$ with both the parallel and antiparallel configurations being low conducting. Again the amplitude of the current variation over $\theta$ increases with bias although only moderately in this case. Furthermore for this situation $v_{\mathrm{d}}$, which is still linear with $V$, is rather sensitive to the angle between the two spins. These differences affect dramatically the macroscopic current, $I$, that we calculate next.

The macroscopic $I-V$ curves for the two cases are presented in the panels (a) and (c) of Fig. 3 while the panels (b) and (d) report the current quadratic fluctuations $\Delta I$ $=\sqrt{\left\langle i^{2}\right\rangle-I^{2}}$ still as a function of bias. In all cases we study the electrical response in the temperature range $1-15 \mathrm{~K}$. The most interesting behavior is found for $\gamma_{C}=1 / 4 \gamma_{L}$, from which we start our discussion.

Figure 3(a) reveals two remarkable features. First we note that there is a pronounced NDC at about 0.2 Volt, which is 


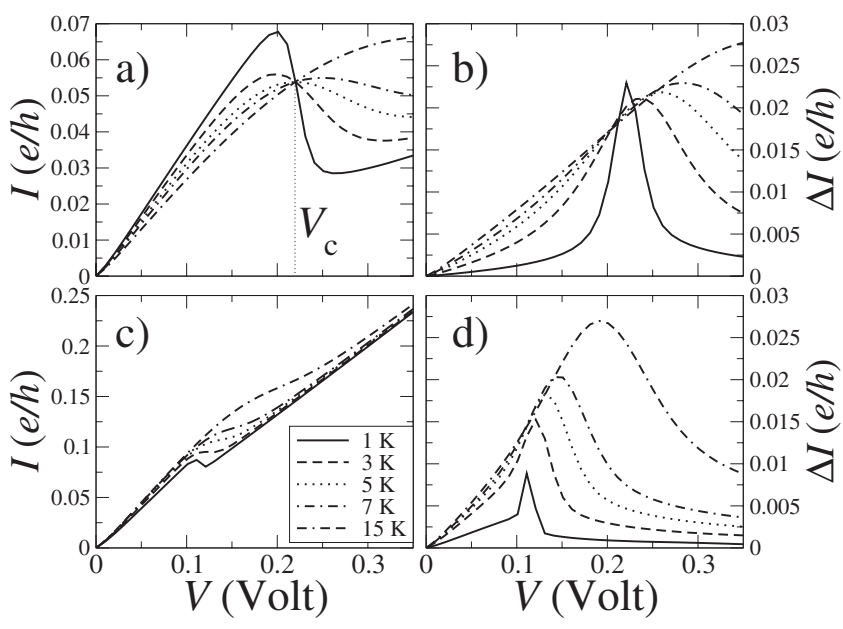

FIG. 3. Macroscopic transport quantities. The $I-V$ curves are presented in panels (a) and (c) while panels (b) and (d) display the current quadratic fluctuations $\Delta I=\sqrt{\left\langle i^{2}\right\rangle-I^{2}}$ as a function of bias $V$. Panels (a) and (b) are for $\gamma_{C}=1 / 4 \gamma_{\mathrm{L}}$ while (c) and (d) are for $\gamma_{\mathrm{C}}$ $=1 / 2 \gamma_{\mathrm{L}}$. Note that in (a) there is a critical voltage $V_{\mathrm{C}}$ at which the current becomes independent of the temperature.

well evident at $1 \mathrm{~K}$, it weakens as the temperature increases and finally disappears at $15 \mathrm{~K}$. Interestingly the scaling of the electrical current with the temperature is opposite at the two sides of the NDC: it decreases as the temperature is enhanced before the NDC while it grows with $T$ for voltages just after the NDC. The same NDC is present also in the case of stronger coupling with the leads $\left[\gamma_{\mathrm{C}}=1 / 2 \gamma_{\mathrm{L}}\right.$, Fig. $\left.3(\mathrm{c})\right]$ at the somewhat lower voltage of about 0.1 Volt. In this case however the NDC is much less pronounced and disappears already at $3 \mathrm{~K}$.

The second and most striking feature of Fig. 3(a) is the presence of a critical voltage, $V_{\mathrm{C}}$, at which the current becomes independent of the temperature. Such a voltage is in the vicinity of the NDC and correlates well with the peak in the current quadratic fluctuations [Fig. 3(b)] at low temperature. Note that this second feature is absent in the case of strong coupling to the leads.

All these aspects can be easily understood by relating the microscopic quantities of Fig. 2 with the average of Eq. (3). Let us consider the case of $\gamma_{C}=1 / 4 \gamma_{\mathrm{L}}$ first. In general the macroscopic current $I(V)$ is determined by the microscopic currents $i(V, \theta)$ of those configurations in which the system spends most of the time. The Eqs. (4) tell us that the ferromagnetic configuration is energetically favorable at low bias while it is the antiferromagnetic to dominate at higher voltages (for $v_{\mathrm{d}}$ larger than $\pm \sqrt{-a / b}$ ). This means that as the external bias increases the average current becomes progressively dominated by antiferromagnetic configurations to the expenses of the ferromagnetic ones. Since the microscopic current for $\theta=\pi$ is always considerably smaller than that for $\theta=0$ [see Fig. 2(a)], this results in a decrease in the macroscopic current as a function of bias, i.e., in the NDC. Note that this particular NDC is not of microscopic electronic origin since the microscopic currents $i(V, \theta)$ are monotonic in $V$ for every $\theta$.

In brief, the NDC described here originates because, as the bias is changed, the system spends on average a different amount of time in the many accessible magnetic configurations. Thus, the present mechanism is different from previously proposed schemes for magnetic molecules. ${ }^{15}$ In that case the NDC is the result of the spin-selection rule forbidden suppression of transitions between different charging states of the molecule. As such the NDC has a microscopic origin and always appears as satellite of the main Coulomb blockade physics, i.e., it strictly occurs when the coupling between the molecule and the electrodes is weak. In contrast our macroscopic NDC is simply rooted in the energetics of the problem and can occur even in the limit of strong coupling and without charging.

The fact that the exchange coupling changes sign as a function of the bias produces the second important feature in the macroscopic $I-V$ curve. In fact when the potential drop between the two magnetic atoms is $v_{\mathrm{d}}= \pm \sqrt{-a / b}$ then the parallel and antiparallel configurations of the magnetic molecule become energetically degenerate. This means that now no magnetic energy scale enters into the problem and the system spends an equal amount of time in any spin configurations regardless of the temperature. In general, $v_{\mathrm{d}}$ is proportional to the external bias $V$. Therefore one expects the existence of a universal external bias $V_{\mathrm{C}}=V\left[\mathcal{E}_{12}\left(v_{\mathrm{d}}\right)=0\right]$ such that $\mathcal{E}_{12}=0$ and the macroscopic currents becomes independent of the temperature as indeed demonstrated in Fig. 3(a). However there is a second condition for this to happen, i.e., $v_{\mathrm{d}}$ should be independent of the angle $\theta$. This is not satisfied for $\gamma_{\mathrm{C}}=1 / 2 \gamma_{\mathrm{L}}$ [see Fig. 2(d)] and as a consequence the $I-V$ curves remain temperature dependent at any bias.

From our discussion it is now clear that if $v_{\mathrm{d}}$ is proportional to $V$ and weakly dependent on $\theta$ then there will be a critical voltage $V_{\mathrm{C}}$ at which any macroscopic quantity becomes temperature independent. Figure 3(a) illustrates this feature for the current and the same is demonstrated in Fig. 4(b) for the conductance $G=\left\langle\frac{\partial i}{\partial V}\right\rangle$. Interestingly one can also adopt a different definition for the macroscopic conductance, namely, that of the bias derivative of the macroscopic current $\partial I / \partial V$. Such a quantity is presented in Fig. 4(a) and as expected it appears sensibly different from $G$. Interestingly both $G$ and $\partial I / \partial V$ are, in principle, accessible from experiments, and one may wonder whether some general conclusions can be taken by measuring the two quantities independently.

In general, by taking the Eq. (3) and formally deriving $Q$ with respect to the bias $V$ we find

$$
\left[\left\langle\frac{\partial q}{\partial V}\right\rangle-\frac{\partial Q}{\partial V}\right] k_{\mathrm{B}} T=\left\langle q \frac{\partial \mathcal{E}_{12}}{\partial V}\right\rangle-Q\left\langle\frac{\partial \mathcal{E}_{12}}{\partial V}\right\rangle,
$$

where $k_{\mathrm{B}}$ is the Boltzman constant. If one now considers $q$ $=i$ then the Eq. (5) establishes a general relation between the conductance fluctuations and the correlation function between the current and the magnetic energy, which reads

$$
\left[G-\frac{\partial I}{\partial V}\right] k_{\mathrm{B}} T=\left\langle i(V, \theta) \frac{\partial \mathcal{E}_{12}(V, \theta)}{\partial V}\right\rangle-I\left\langle\frac{\partial \mathcal{E}_{12}(V, \theta)}{\partial V}\right\rangle .
$$

Such a relation is drastically simplified when the microscopic current has the typical spin-valve dependence $i(V, \theta)$ 

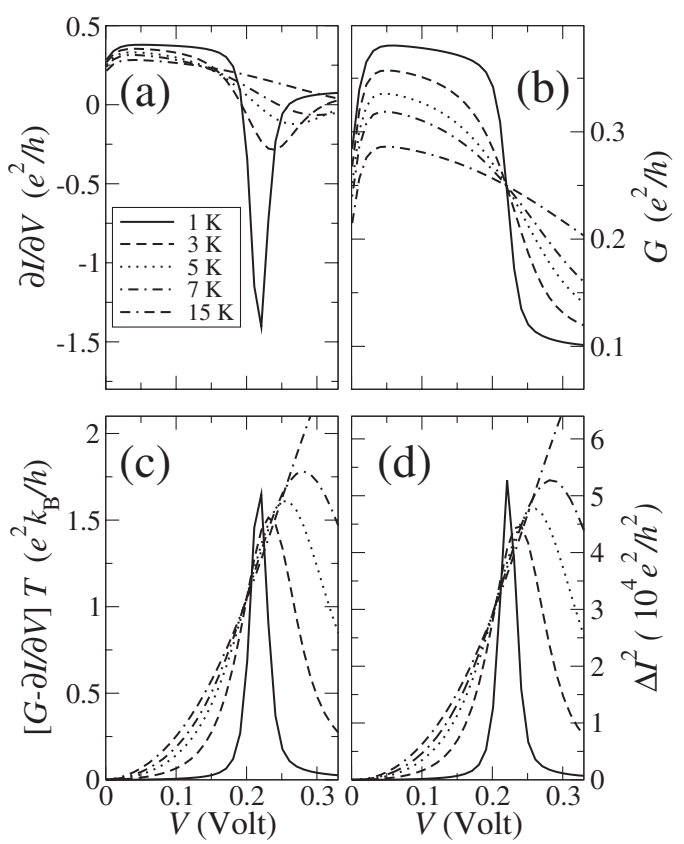

FIG. 4. Differential conductance calculated as (a) the derivative of the macroscopic current, $I$, with respect to the bias or as the (b) thermal average, $G$, of the microscopic conductance. In panel (c) we show the product of $(G-\partial I / \partial V)$ with the temperature $T$ as a function of bias. Results are presented for $\gamma_{C}=1 / 4 \gamma_{\mathrm{L}}$. Note that $[G-\partial I / \partial V] T$ is proportional to the square of the current quadratic fluctuations $\Delta I^{2}$ [panel (d)] of Fig. 3(b).

$\sim\left[i_{0}+i_{1} \cos \theta\right] V$ and $v_{\mathrm{d}}$ is linear with $V$. In this situation (encountered here for $\gamma_{\mathrm{C}}=1 / 4 \gamma_{\mathrm{L}}$ ) one finds

$$
\left[G-\frac{\partial I}{\partial V}\right] k_{\mathrm{B}} T \propto \Delta I^{2},
$$

i.e., that the conductance fluctuations rescaled by the temperature are proportional to the squared current fluctuations. A numerical proof of such a relation is provided in the panels (c) and (d) of Fig. 4, where we show, respectively, $G$ $-\partial I / \partial V$ and $\Delta I^{2}$.

We believe that such a relation can be verified experimentally. The right-hand side of Eq. (7) is essentially the square of the current noise and it is directly addressable experimentally. The key point is then that of evaluating the left-hand side, i.e., of measuring independently $G$ and $\partial I / \partial V$. The second quantity $(\partial I / \partial V)$ is simply the numerical derivative of the $I-V$ curve measured by slowly sweeping the bias in a dc measurement. In contrast we can extract $G$ from the real part of the complex conductivity obtained as the linear response to a slow ac modulation around a given voltage $V$. Note also that our argument holds if the dominant fluctuations of the current have the magnetic origin discussed here. Therefore we speculate that this relation can be verified in a temperature range where vibrational fluctuations are suppressed and where quantum tunneling is still negligible.

Before concluding we wish to point out that our main result, namely, the existence of a critical voltage $V_{C}$ at which the macroscopical observable become temperature independent, is expected to hold valid even to a fully quantummechanical description of the molecule spin. In fact the only two conditions for our argument to be valid are that there must exist two different spin states of the molecule having different scattering properties ${ }^{16}$ and that these two states must become energetically degenerate for a certain value of the external bias potential. These two conditions can be certainly met even if the molecule spins are treated quantum mechanically. However, in the quantum case we expect quantum tunneling to dominate the transition between the two spin states at low temperature and voltages near $V_{\mathrm{C}}$ (i.e., when the two states are quasidegenerate). In that situation low-temperature anomalies in the $I-V$ around $V_{\mathrm{C}}$ are likely to be present. These cannot be described by our classical model.

\section{CONCLUSION}

In conclusion we have investigated the temperaturedependent electronic transport through a model diatomic magnetic molecule, in which the exchange coupling between the two magnetic centers is a function of the bias. This presents two remarkable characteristics. First, if the potential drop between the two magnetic centers is only weakly dependent on the angle between their magnetic moments and it is linear in $V$ then there is a critical voltage $V_{\mathrm{C}}$ at which the macroscopic current becomes temperature independent. Second, if in addition the microscopic current has a form $i(V, \theta) \sim\left[i_{0}+i_{1} \cos \theta\right] V$ then there is a universal relation between the temperature-rescaled conductance fluctuations and the quadratic current fluctuations. Both these effects are a unique fingerprint of the dependence of the magnetic energy upon an external bias and can be used as a tool for detecting such a dependence.

\section{ACKNOWLEDGMENTS}

This work is funded by Science Foundation of Ireland. We thank Maria Stamenova and Chaitanya Das Pemmaraju for helping with the numerical implementation, and Tchavadar Todorov for useful discussion.

\footnotetext{
*Present address: Department of Physics, Indian Institute of Technology Delhi, New Delhi 110 016, India.

†sanvitos@tcd.ie

${ }^{1}$ S. Katano, Y. Kim, M. Hori, M. Trenary, and M. Kawai, Science 316, 1883 (2007).
}

${ }^{2}$ B. J. McIntyre, M. Salmeron, and G. A. Somorjai, Science 265, 1415 (1994).

${ }^{3}$ M. N. Baibich, J. M. Broto, A. Fert, F. Nguyen Van Dau, F. Petroff, P. Etienne, G. Creuzet, A. Friederich, and J. Chazelas, Phys. Rev. Lett. 61, 2472 (1988) ; G. Binasch, P. Grünberg, F. 
Saurenbach, and W. Zinn, Phys. Rev. B 39, 4828 (1989).

${ }^{4}$ J. Slonczewski, J. Magn. Magn. Mater. 159, L1 (1996).

${ }^{5}$ C. F. Hirjibehedin, C.-Y. Lin, A. F. Otte, M. Ternes, C. P. Lutz, B. A. Jones, and A. J. Heinrich, Science 317, 1199 (2007).

${ }^{6}$ L. Bogani and W. Wernsdorfer, Nature Mater. 7, 179 (2008).

${ }^{7}$ N. Baadji, M. Piacenza, T. Tugsuz, F. Della Sala, G. Maruccio, and S. Sanvito, Nature Mater. 8, 813 (2009).

${ }^{8}$ M. Diefenbach and K. S. Kim, Angew. Chem., Int. Ed. 46, 7640 (2007).

${ }^{9}$ J. Lehmann, A. Gaita-Ario, E. Coronado, and D. Loss, Nat. Nanotechnol. 2, 312 (2007).

${ }^{10}$ G. A. Timco, S. Carretta, F. Troiani, F. Tuna, R. J. Pritchard, C. A. Muryn, E. J. L. McInnes, A. Ghirri, A. Candini, P. Santini, G.
Amoretti, M. Affronte, and R. E. P. Winpenny, Nat. Nanotechnol. 4, 173 (2009).

${ }^{11}$ K. Yosida, Theory of Magnetism (Springer-Verlag, Berlin, 1996).

${ }^{12}$ M. Stamenova, T. N. Todorov, and S. Sanvito, Phys. Rev. B 77, 054439 (2008).

${ }^{13}$ S. Datta, Electronic Transport in Mesoscopic Systems (Cambridge University Press, Cambridge, 1995).

${ }^{14}$ A. R. Rocha and S. Sanvito, Phys. Rev. B 70, 094406 (2004).

${ }^{15}$ H. B. Heersche, Z. de Groot, J. A. Folk, H. S. J. van der Zant, C. Romeike, M. R. Wegewijs, L. Zobbi, D. Barreca, E. Tondello, and A. Cornia, Phys. Rev. Lett. 96, 206801 (2006).

${ }^{16}$ C. D. Pemmaraju, I. Rungger, and S. Sanvito, Phys. Rev. B 80, 104422 (2009). 